\title{
"Click" patterning of self-assembled monolayers on hydrogen-terminated silicon surfaces and their characterization using light-addressable potentiometric sensors
}

Jian Wang, Fan Wu, Michael Watkinson ${ }^{1}$, Jingyuan Zhu', Steffi Krause*

School of Engineering and Materials Science, Queen Mary University of London, Mile End Road, London E1 4NS, UK

${ }^{1}$ School of Biological and Chemical Sciences, Queen Mary University of London, Mile End Road, London E1 4NS, UK

*Corresponding author: s.krause@qmul.ac.uk, Telephone +44 (0)2078823747

\section{Abstract}

Two potential strategies for chemically patterning alkyne-terminated self-assembled monolayers (SAMs) on oxide-free silicon or silicon-on-sapphire (SOS) substrates were investigated and compared. The patterned surfaces were validated using a light-addressable potentiometric sensor (LAPS) for the first time. The first strategy involved an integration of photolithography with "click" chemistry. Detailed surface characterization (i.e. water contact angle, ellipsometry, AFM and XPS) and LAPS measurements showed that photoresist processing not only decreases the coverage of organic monolayers, but also introduces chemically bonded contaminants on the surfaces, thus significantly reducing the quality of the SAMs and the utility of "click" surface modification. The formation of chemical contaminants in photolithography was also observed on carboxylic acid and alkyl terminated monolayers using LAPS. In contrast, a second approach combined microcontact printing $(\mu \mathrm{CP})$ with "click" chemistry, that is, azide (azido-oligo(ethylene glycol) $(\mathrm{OEG})-\mathrm{NH}_{2}$ ) inks were printed on alkyne-terminated SAMs on silicon or SOS through PDMS stamps. The surface characterization results for the sample printed with a flat featureless PDMS stamp 
demonstrated a non-destructive and efficient method of $\mu \mathrm{CP}$ to perform "click" reactions on alkyne terminated, oxide-free silicon surfaces for the first time. For the sample printed with a featured PDMS stamp, LAPS imaging showed a good agreement with the pattern of the PDMS stamp, indicating the successful chemical patterning on non-oxide silicon and SOS substrates and the capability of LAPS to image the molecular patterns with high sensitivity.

\section{Introduction}

The patterning of chemical and biochemical molecules on silicon surfaces has become an important feature in label-free biosensing and cell tissue engineering applications ${ }^{1-2}$. Selfassembled monolayers (SAMs), as ultrathin resists on silicon surfaces, provide an effective platform for such chemical patterning. A variety of techniques have been developed for the formation of patterned SAMs on silicon ${ }^{3-5}$. The most popular methods are photolithography and microcontact printing $(\mu \mathrm{CP})$, which have both been used to fabricate patterns with various molecules ${ }^{6-11}$ or biological components (i.e. DNA ${ }^{12-16}$ and peptides ${ }^{17-20}$ ).

The concept of "click" chemistry was first described by Sharpless' group in $2001^{21}$ and covered a wide range of chemical reactions. An ideal "click" reaction occurs under very mild conditions with high yield and with minimal by-products. Without doubt, the most welldocumented reaction within the suite of "click" reactions is the copper(I)-catalyzed azide alkyne cycloaddition (CuAAC) reaction. Recently, the CuAAC reaction has been frequently applied to the surface modification of silicon ${ }^{22-24}$ and allows engineering the architecture and function of materials in an efficient and modular way. Therefore, through the combination of "click" chemistry and patterning techniques, chemically patterned silicon surfaces can be readily fabricated. In many cases, organic monolayers on silicon have been patterned by selective removal of parts of the monolayer by exposure to an electron beam ${ }^{25}, \mathrm{UV}^{26}$ or visible light $^{27}$ using the monolayer as resist or by light induced deposition on silicon 
surfaces $^{28}$. In many biosensing or electronic device applications, a perfectly passivating monolayer is required, in which case patterning by photolithography employing a commercial photoresist would be desirable. However, as a result of the high solubility of positive photoresist in most organic solvents, the combined technique of "click" chemistry and photolithography could only be carried out in aqueous solutions, which may limit the application of this method. Zhu et al. have reported a strategy for chemically patterning 1, 8nonadiyne monolayer modified porous silicon with two different species (-OEG-OCH OEG-OH) using photolithography with a positive photoresist followed by a surface modification using "click" chemistry ${ }^{6-7}$. Initially, a metal film was pre-coated on the 1,8 nonadiyne monolayer modified surface in order to exclude the photoresist from the silicon pores, although, the metal film was found to have an adverse effect on the efficiency of the "click" reaction and the metal layer was consequently eliminated". To the best of our knowledge, there have been no reports on the effects of photoresist exposure and development on the integrity of SAMs on silicon substrates and the efficacy of their further chemical modification. As an alternative strategy, Ravoo and Reihoudt have elegantly demonstrated that a "click" reaction can be also performed via $\mu \mathrm{CP}$ within a short reaction time, thus providing a straightforward way for the fabrication of biomolecular microarrays on silicon ${ }^{9,14,29}$. A siloxane monolayer ( $\mathrm{Si}-\mathrm{O}-\mathrm{Si}-\mathrm{R}$ ) was used as the platform for patterning, however, it was reported that this monolayer was inherently unstable and prone to hydrolytic cleavage in electrolyte solutions ${ }^{30}$. This resulted in an additional silicon dioxide layer which adversely affects the sensitivity of silicon based bioelectronics devices and biosensors ${ }^{31}$. Thus, if this method is to find utility in the fabrication of chemical patterns on oxide-free silicon surfaces in these applications, it is essential to eliminate the intervening layer of silicon $\operatorname{dioxide}^{30}$. Kehr et al. have shown that a "click" reaction via $\mu \mathrm{CP}$ is also applicable on SAMs of nanoparticles, providing a strategy for cell adhesion and cellular patterning ${ }^{32}$. 
Light-addressable potentiometric sensors (LAPS), which are based on an electrolyte/insulator/silicon (EIS) field-effect structure, can record both surface potentials and impedance with spatial resolution ${ }^{33-34}$. They have been widely used as both biochemical sensors $^{35-37}$ and cell-based biosensors ${ }^{38-42}$. We have recently demonstrated that SAMs can be used as the insulator in LAPS measurements and that this significantly improves the sensitivity of LAPS compared to a traditional silicon dioxide insulator ${ }^{33}$. High-resolution LAPS imaging with SAM modified silicon-on-sapphire (SOS) substrates was validated using micropatterns of polyelectrolyte/DNA that were physically adsorbed on the sensor surface ${ }^{33}$.

As a result of these recent advances, we wished to exploit the chemical patterning strategy on SAM modified oxide-free silicon surfaces in order to image cell-surface interactions on patterned surfaces using LAPS. 1, 8-Nonadiyne, which is commercially available, has been used extensively for the formation of high-quality monolayers on oxide-free silicon ${ }^{6-7,23,43-46}$. The resultant alkyne-terminated surface provides an ideal platform for copper catalyzed azide-alkyne cycloadditions (CuAAC) "click" functionalization ${ }^{6-7,23,43-46}$, which attracted us to apply it in patterning of a non-oxidized Si/SOS surface. Herein we report a comparison of the chemical patterning methods of photolithography and $\mu \mathrm{CP}$ on non-oxidized silicon surfaces integrated with "click" chemistry together with LAPS imaging to validate the molecular patterning.

\section{Experimental section}

2.1 Materials. Silicon-on-sapphire (SOS) with a $1 \mu \mathrm{m}$ thick silicon (100) layer (boron doped, $0.1 \Omega \cdot \mathrm{cm}$ ) on a $475 \mu \mathrm{m}$ thick sapphire substrate was purchased from Monocrystal, Russia. Double polished silicon (100) (boron doped, 10-30 $\Omega \cdot \mathrm{cm}$ ) was purchased from Si-MAT, Germany. All chemicals and reagents, unless otherwise noted, were purchased from SigmaAldrich. The following reagents were used as received: hydrogen peroxide solution $(30 \mathrm{wt} . \%$ 
in $\mathrm{H}_{2} \mathrm{O}$, semiconductor grade), sulfuric acid (95.0-98.0\%, semiconductor grade), hydrochloride acid (ACS regent), ethanol (100\%), sodium ascorbate (98\%), copper(II) sulfate pentahydrate $(99 \%), N, N, N^{\prime}, N^{\prime}$--tetramethylethylenediamine (TMEDA, $\left.\geq 99.5 \%\right)$ and 11 azido-3,6,9-trioxaundecan-1-amine (azido-OEG- $\mathrm{NH}_{2}, \quad$ technical, $\geq \quad \geq 90 \%$ (GC)). Dichloromethane (DCM) used for cleaning was redistilled prior to use. 1, 8-Nonadiyne (98\%) was redistilled from sodium borohydride (99+\%) and stored under $\operatorname{argon}^{45,47}$. The photoresist S1818 and Microposit 351 were purchased from Shipley, UK. A Sylgard 184 poly(dimethyl siloxane) (PDMS) kit was purchased from Dow Corning. For photocurrent measurements, $10 \mathrm{mM}$ phosphate buffer solution $\mathrm{pH} 7.4$ containing $137 \mathrm{mM} \mathrm{NaCl}$ and $2.7 \mathrm{mM} \mathrm{KCl}$ was used. All solutions were prepared with water $(18.2 \Omega \cdot \mathrm{cm})$ from a three stage Millipore MilliQ 185 water purification system (Millipore, USA). Argon was dried and purified through an oxygen/moisture trap (Agilent Technologies, USA).

2.2 Assembly of 1, 8-nonadiyne monolayer and CuAAC “click" derivatization. Siliconon-sapphire (SOS) wafers and double polished silicon wafers were cut into $7 \mathrm{~mm} \times 7 \mathrm{~mm}$ pieces. In order to form the ohmic contact for LAPS measurements, $30 \mathrm{~nm} \mathrm{Cr}$ and $150 \mathrm{~nm} \mathrm{Au}$ were thermally evaporated onto one corner of the chip and subsequently heated to $300{ }^{\circ} \mathrm{C}$ for 5 min as previously reported ${ }^{33}$. The substrate was cleaned in a hot piranha solution $13: 1$ $\mathrm{H}_{2} \mathrm{SO}_{4}(96 \%) / \mathrm{H}_{2} \mathrm{O}_{2}(30 \%)$, caution: highly corrosive) at $100{ }^{\circ} \mathrm{C}$ for $30 \mathrm{~min}$ and then rinsed copiously with ultrapure (Milli-Q) water. The assembly of the 1, 8-nonadiyne monolayer followed the procedure reported by Ciampi et al. ${ }^{45,47}$. The cleaned SOS or silicon sample was transferred to a $2.5 \% \mathrm{HF}$ solution and chemically etched for $90 \mathrm{~s}$ to obtain the H-terminated surface (caution: HF is highly corrosive). AFM measurements of the hydrogen terminated silicon surface confirmed a surface roughness of $0.2 \mathrm{~nm}$, which is similar to the one reported for a good quality $\operatorname{Si}(100)$ surface $^{48}$. During the cleaning and etching time, the redistilled 1,8 nonadiyne was transferred into a Schlenk tube and was degassed by freeze-pump-thaw cycles 
until no gas bubbles evolved from the solution. Then the freshly prepared H-SOS or H-Si sample was transferred into the degassed 1, 8-nonadiyne and left for $3 \mathrm{~h}$ at $165^{\circ} \mathrm{C}$ under an argon stream. After cooling to room temperature, the functionalized surface (surface 1, Scheme 1a) was then rinsed with copious amounts of redistilled DCM and blown dry with nitrogen.

The alkyne-terminated surface was further functionalized trough a typical "click" procedure (surface 2, Scheme 1a). The substrate was transferred into a vessel containing the "click" solution of the azido-OEG-NH $\mathrm{H}_{2} \quad(15 \mathrm{mM}$, ethanol/water 2:1), copper(II) sulfate pentahydrate $(1.1 \mathrm{~mol} \%$ relative to the azide), sodium ascorbate (10 mol \% relative to the azide) and TMEDA $(0.45 \mathrm{mM})$. The reaction was carried out in the dark at room temperature for $24 \mathrm{~h}$. The unreacted reagents were removed by rinsing the substrate consecutively with copious amounts of ethanol, water and ethanol. Then the sample was placed in a $0.5 \mathrm{M}$ hydrochloric acid solution for 2 min to remove the residual copper ${ }^{44}$. Finally it was washed with copious amounts of water and ethanol.

\subsection{Photolithography on 1, 8-nonadiyne monolayer modified surface and "click"} reaction. S1818 patterns were formed on surface 1 with an optimized photolithography process. First, S1818 was spin-coated onto a 1, 8-nonadiyne modified substrate at $500 \mathrm{rpm}$ for $5 \mathrm{~s}$ and then $5000 \mathrm{rpm}$ for $60 \mathrm{~s}$. After spin-coating, the film was baked at $95{ }^{\circ} \mathrm{C}$ for $15 \mathrm{~min}$ on a hot plate. Then it was exposed to UV light for $20 \mathrm{~s}$ through a mask (Scheme 1b, route ii) or was exposed over the entire surface (Scheme 1b, route iii) using a KARL SUSS MJB3 UV400 mask aligner. The substrate was then developed in diluted Microposit 351 solution (Microposit 351/water 1:3) for $45 \mathrm{~s}$ and washed with pure water and blown dry. To study the effect of photolithography on SAMs, the substrate was then ultrasonically washed with acetone for 5 min to remove any photoresist and then rinsed with isopropanol and pure water. 
The UV exposed parts of surface 3 are therefore identical to surface 4 . Surface 4 was transferred into a "click" solution as described in Section 2.2 to produce surface 5 .

2.4 Microcontact printing $(\boldsymbol{\mu C P})$ for "click" chemistry. The master and PDMS stamps were prepared following a previously reported protocol ${ }^{49,33}$. The pattern of the PDMS stamp consisted of circular islands with a diameter of $40 \mu \mathrm{m}$ and $30 \mu \mathrm{m}$ gaps. Scheme 1c illustrates the process of "click" chemistry on surface 1 using $\mu \mathrm{CP}$. The PDMS stamp was dipped in the “click" solution for $30 \mathrm{~min}$. The soaked stamp was dried under nitrogen gas and placed onto the alkyne-terminated surface for $3 \mathrm{~h}$ at room temperature. The reaction was performed in a closed Petri dish with a moistened tissue. After removing the stamp, the unreacted reagent was removed by rinsing the substrate consecutively with copious amounts ethanol, water and ethanol. Then the sample was immersed in a $0.5 \mathrm{M}$ hydrochloric acid solution for $2 \mathrm{~min}$ to remove the copper residue and rinsed with copious amounts water ${ }^{44}$. To characterize the “clicked" surface properly, a chemically homogeneous surface (surface 7) was also prepared using a flat PDMS stamp following the same procedure. 


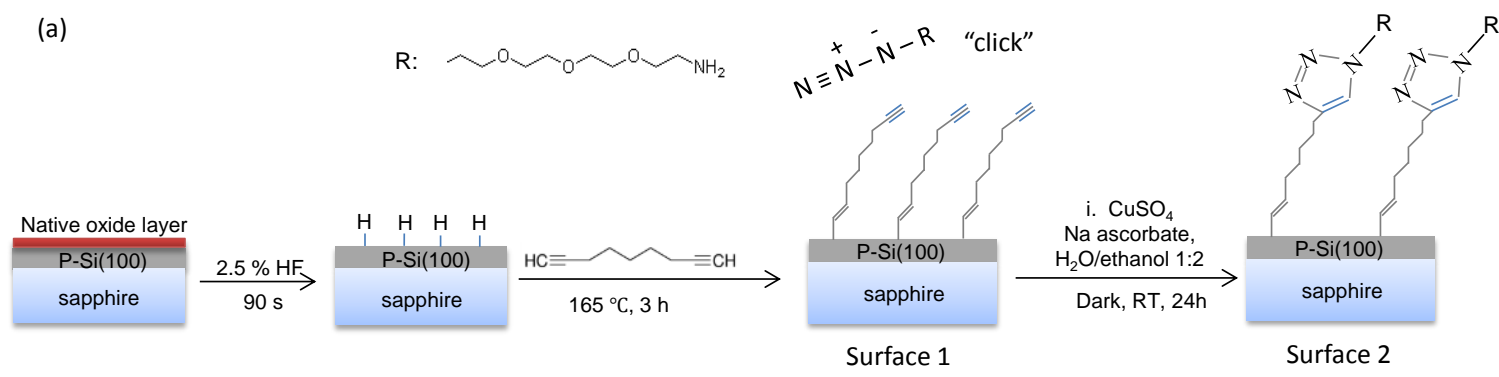

(b)

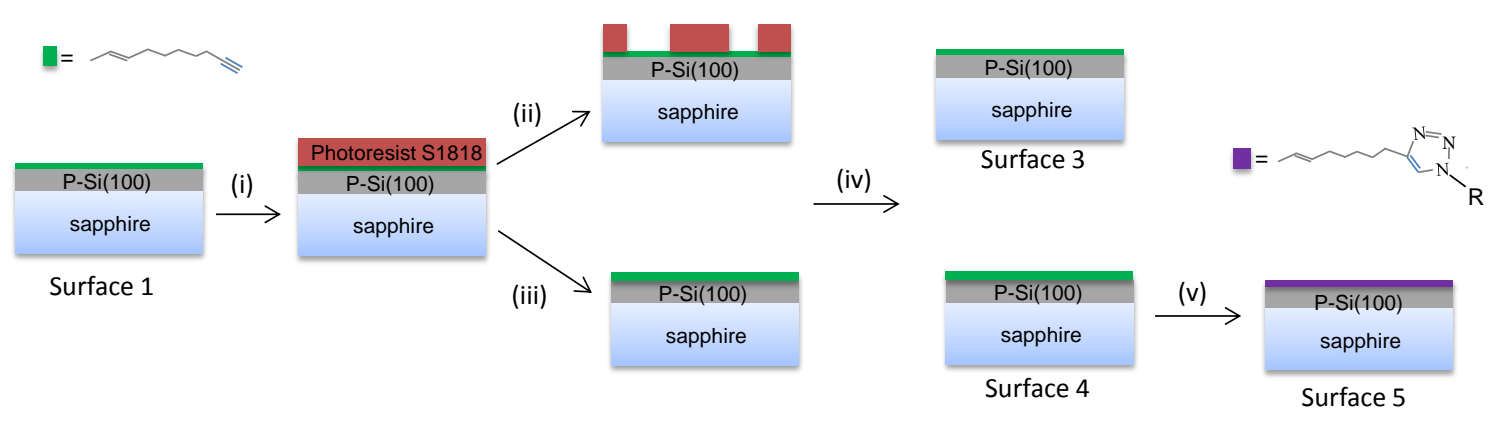

(c)
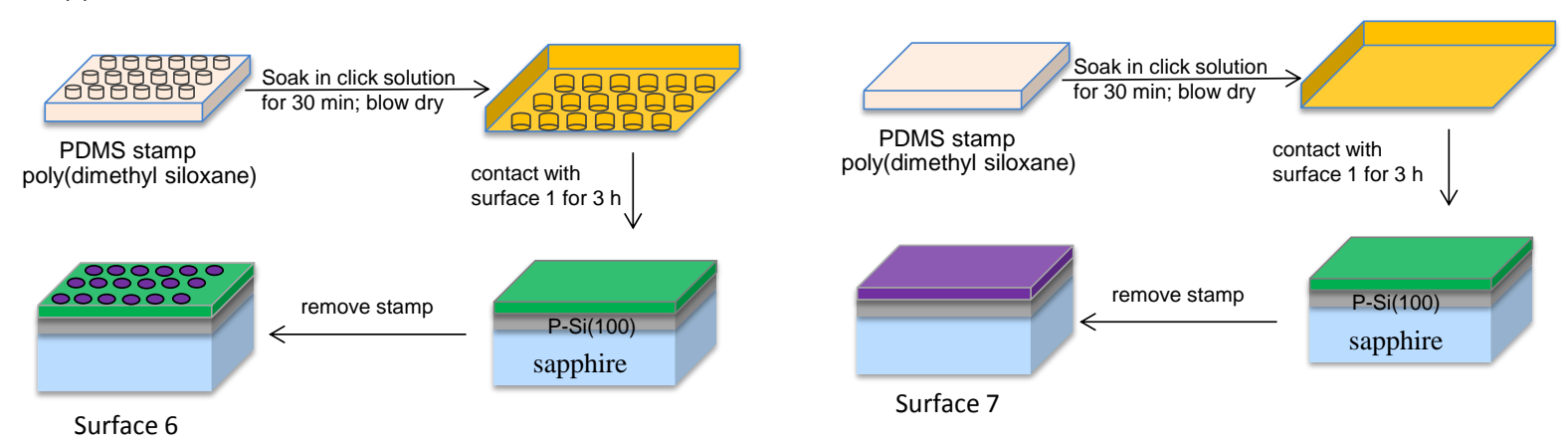

Scheme 1. (a) Modification of 1, 8-nonadiyne monolayers on SOS substrates (surface 1) and attachment of azido-OEG-NH${ }_{2}$ using "click" chemistry (surface 2) (b) procedure for photolithography on surface 1 and "click" chemistry on the surface after photolithography: i) S1818 photoresist was spin-coated on surface 1; ii) S1818 coated substrate was exposed to UV light through a mask and then developed in diluted Microposit 351 solution; the mask used was either structured with stripes of $20 \mu \mathrm{m}$ width (UV-blocked region) and $10 \mu \mathrm{m}$ interspace (UV-exposed region) or as shown in Scheme S1b (Supporting Information); iii) the entire S1818 coated surface was exposed with UV light and then developed in diluted Microposit 351 solution; iv) samples were ultrasonically washed in acetone and isopropanol 
to remove the cured photoresist to obtain surfaces 3 and 4; v) azido-OEG- $\mathrm{NH}_{2}$ was attached onto surface 4 via "click" chemistry (c) schematic illustration of the $\mu \mathrm{CP}$ chemistry: azidoOEG-NH $\mathrm{NH}_{2}$ was printed on surface 1 and "click" reactions occurred exclusively in the contact area (surface 6: an array of chemical pattern, surface 7: a homogeneous surface functionalized with azido-OEG-NH $\mathrm{N}_{2}$ ). For ellipsometry and AFM measurements, the above surface modification was carried out on silicon substrates.

2.5 Surface characterization. The water wettability of monolayer modified SOS samples were measured using a Drop Shape Analysis System (Krüss DSA100, Germany). $1 \mu \mathrm{L}$ of ultrapure water was carefully deposited onto the surface and three spots were measured on each sample and averaged.

An alpha-SE ${ }^{\circledR}$ Spectroscopic Ellipsometer (J.A. Woollam Co. Inc., USA) and CompleteEASE software (J.A. Woollam Co. Inc., USA) for data collection and analysis were used to measure the thickness of monolayers on silicon surfaces. A He-Ne laser $(632.8 \mathrm{~nm})$ and an angle of incidence of $70^{\circ}$ were adopted.

X-ray photoelectron spectroscopy (XPS) experiments were performed on modified SOS surfaces at the nanoLAB of Newcastle University, UK, using a Kratos Axis Nova spectrometer with CasaXPS software. Survey scans were carried out over a $1100 \sim 0 \mathrm{eV}$ range with a $1.0 \mathrm{eV}$ step size, a $100 \mathrm{~ms}$ dwell time, and an analyzer pass energy of $100 \mathrm{eV}$. Highresolution scans were run with a $0.1 \mathrm{eV}$ step size, a dwell time of $100 \mathrm{~ms}$, and the analyser pass energy set to $20 \mathrm{eV}$. The scan regions were Si $2 \mathrm{p}(97-107 \mathrm{eV}), \mathrm{C} 1 \mathrm{~s}(278-294 \mathrm{eV}), \mathrm{N} 1 \mathrm{~s}$ (392-408 eV), O 1s (526-542 eV) and $\mathrm{Cu} 2 \mathrm{p}_{3 / 2}(926-938 \mathrm{eV})$.

Atomic Force Microscopy (AFM, Dimension Icon, Bruker, US) in tapping mode was used to image the patterns on the Si surface. A NCHV-A (Silicon, $40 \mathrm{~N} / \mathrm{m}$, Al reflective coating, Bruker) tip was used for scanning. 
2.6 LAPS setup. The experimental setup for photocurrent measurements (Figure S1, Supporting Information) has been described elsewhere ${ }^{50}$. In brief, a laser diode module LD1539 (Laser 2000, $\lambda=405 \mathrm{~nm}, 1 \mathrm{~mW}$ ) was modulated electronically at $1 \mathrm{kHz}$ and focused onto the silicon layer using an LD Plan-NEOFLUAR 40x objective with correction ring (Zeiss, numerical aperture 0.6). The sample holder was mounted onto an M-VP-25XL XYZ positioning system (Newport, UK). AC photocurrents were measured using an EG\&G 7260 lock-in amplifier. In all measurements, SOS substrates were used as the working electrode. A platinum electrode with a serial capacitor of $4.7 \mu \mathrm{F}$ and a $\mathrm{Ag} / \mathrm{AgCl}(3 \mathrm{M} \mathrm{KCl})$ electrode in parallel were used as the counter and reference electrodes. The control software was written in LabView ${ }^{50}$.

\section{Results and discussion}

Assembly of SAMs from 1, 8-nonadiyne and "click" functionalization. 1, 8-Nonadiyne monolayers were characterized by water contact angle, ellipsometry and XPS. As shown in Table 1 , the water contact angle for an alkyne-terminated surface $\left(88 \pm 1^{\circ}\right)$ is indicative of a hydrophobic monolayer, as expected, and is comparable to literature results ${ }^{43,45,47}$. The ellipsometric thickness of the 1, 8-nonadiyne monolayer on the silicon substrate was $10.6 \AA$, which is smaller than the calculated value $(12.2 \AA)$, indicative of a tilt angle of $\sim 30^{\circ}$ between the axis of the molecules and the surface normal. XPS spectra of the modified SOS surface (Figure 1a) showed the presence of $\mathrm{Si}, \mathrm{C}$, and $\mathrm{O}$, which is in good agreement with results published previously ${ }^{43,45,47} 45$. The high-resolution narrow scans of XPS provide information on bonding configurations. The $\mathrm{C} 1 \mathrm{~s}$ narrow scan included a main $\mathrm{C}-\mathrm{C}$ peak $(\sim 285.0 \mathrm{eV})$, and two small peaks from $\mathrm{Si}-\mathrm{C}=\mathrm{C}(\sim 284.1 \mathrm{eV})$ and $\mathrm{C}-\mathrm{O}(286.4 \mathrm{eV})$ (Figure 1b), the latter attributed to adventitious covalently bound oxygen-bond contaminants, presumably originating from the 1, 8-nonadiyne $\mathrm{e}^{43,51}$. The binding energies observed were consistent with the results reported elsewhere ${ }^{24,43,51}$. The absence of a Si-O peak in the 101-104 eV region of the high-resolution Si $2 p$ scan indicated good quality monolayers that can effectively protect the underlying silicon substrate from oxidation ${ }^{45,47,52}$ (Figure 1c). 
Table 1. Contact angles and ellipsometry thicknesses of different surfaces

\begin{tabular}{ccc}
\hline Surface & Water contact angle $\left({ }^{\circ}\right)$ & Ellipsometry thickness $(\AA)$ \\
\hline 1 & $88 \pm 1$ & $10.6 \pm 0.4$ \\
2 & $37 \pm 1$ & $16.0 \pm 1.0$ \\
4 & $76 \pm 2$ & $15.5 \pm 0.3$ \\
5 & $52 \pm 4$ & $21.4 \pm 1.2$ \\
7 & $46 \pm 2$ & $15.8 \pm 0.9$
\end{tabular}
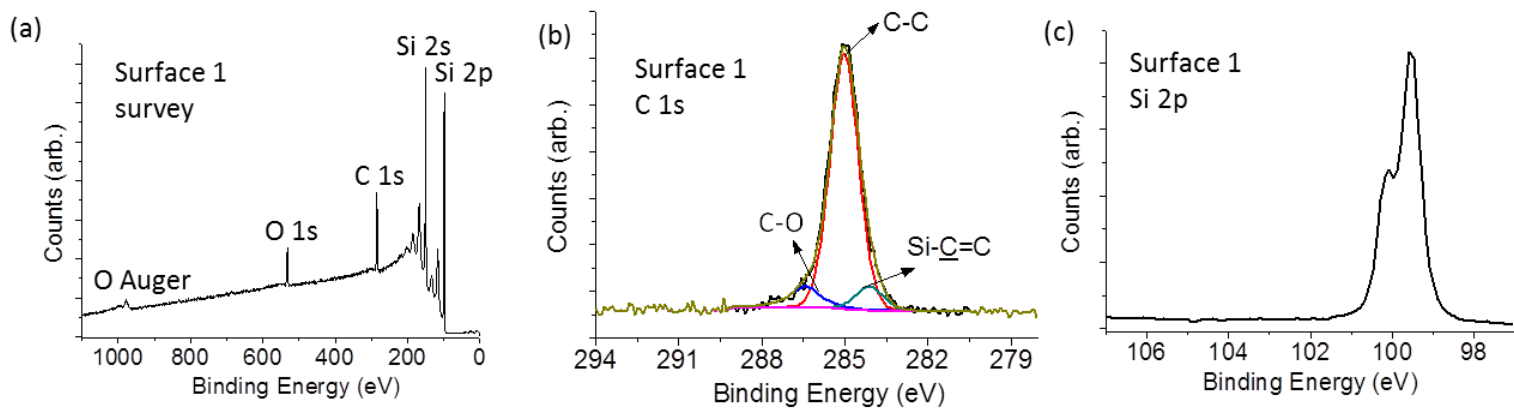

Figure 1. (a) XPS survey scan of 1, 8-nonadiyne monolayer modified SOS. High resolution scans for (b) carbon and (c) silicon.

The alkyne-terminated organic monolayers on non-oxidized silicon surfaces provide a chemical handle for further functionalization via CuAAC "click" chemistry. Due to the presence of OEG and amino moieties, the contact angle for the "clicked" surface (surface 2) decreased dramatically by $\sim 51^{\circ}$ (Table 1 ), which is consistent with previously reported values44, 53. The ellipsometric result shows that after the "click" reaction, the thickness of the monolayer increased to $\sim 16.0 \AA$, which is comparable to the thickness of $17.3 \AA$ determined from X-ray reflectivity (XRR) analysis44. The successful "click" reaction was also demonstrated by XPS results. The XPS survey spectrum (Figure 2a) shows an N 1s peak at $\sim 401 \mathrm{eV}$, indicative of a triazole formation. The absence of $\mathrm{Cu} 2 \mathrm{p} 3 / 2$ emission at $\sim 933 \mathrm{eV}$ indicates no residual copper catalyst on the surface. The narrow scan signal of the $\mathrm{N} 1 \mathrm{~s}$ region (Figure $2 \mathrm{~b}$ ) was fitted to two peaks at $400.6 \mathrm{eV}$ and $402.2 \mathrm{eV}$, which were assigned to 
$\mathrm{C}-\underline{\mathrm{NH}}_{2} / \mathrm{N}-\underline{\mathrm{N}}=\underline{\mathrm{N}}$, and $\underline{\mathrm{N}}-\mathrm{N}=\mathrm{N}$, respectively ${ }^{44,54-55}$. The ratio of the integrated areas $(\sim 2.4: 1)$ is smaller than the stoichiometric ratio of $3: 1$ but is comparable to the literature result $(\sim 2.6: 1)^{44}$. The narrow scan from the $\mathrm{C} 1 \mathrm{~s}$ region was deconvoluted into three peaks assigned to $\mathrm{Si}-\underline{\mathrm{C}}=\mathrm{C}$ $(284.3 \mathrm{eV}), \mathrm{C}-\mathrm{C}(285.3 \mathrm{eV})$ and $\underline{\mathrm{C}}-\mathrm{N} /-\mathrm{O}(287.1 \mathrm{eV})^{44,56}$. Importantly, no $\mathrm{SiO}_{\mathrm{x}}$ species was observed in the Si $2 p$ narrow scan, indicating that a high quality functionalized surface has been prepared (Figure 2d).

(a)

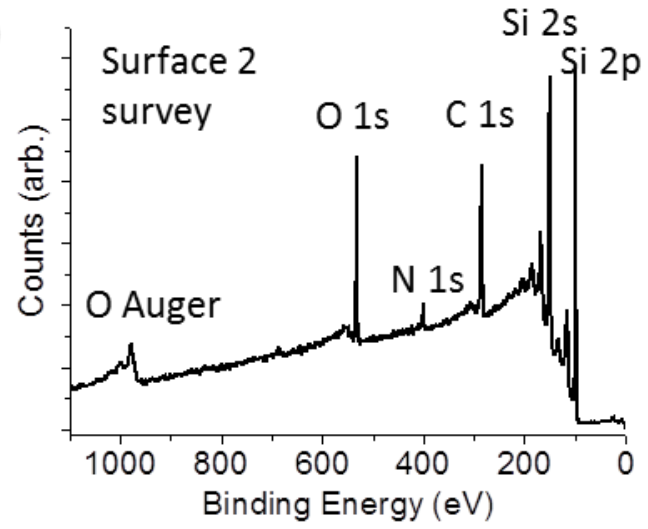

(c)

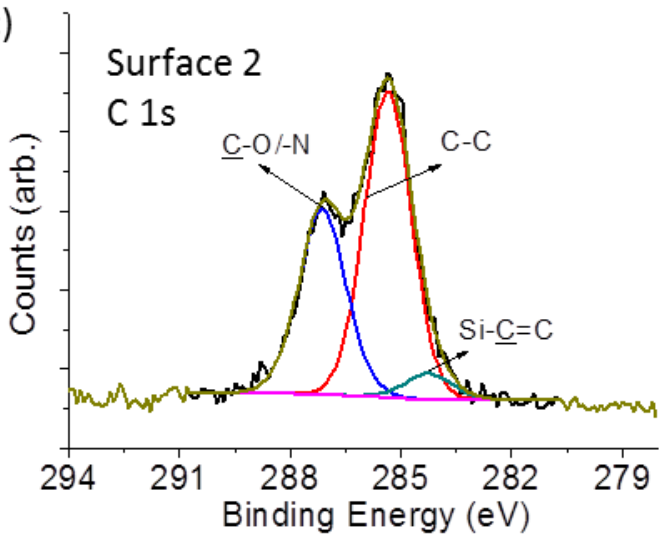

(b) - Surface 2

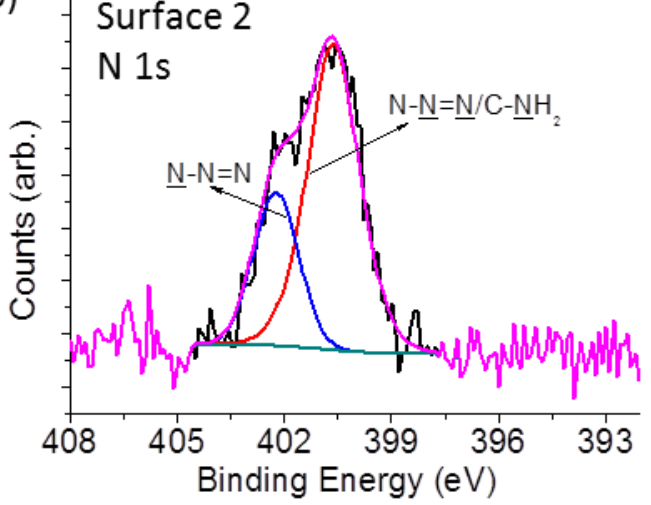

(d)

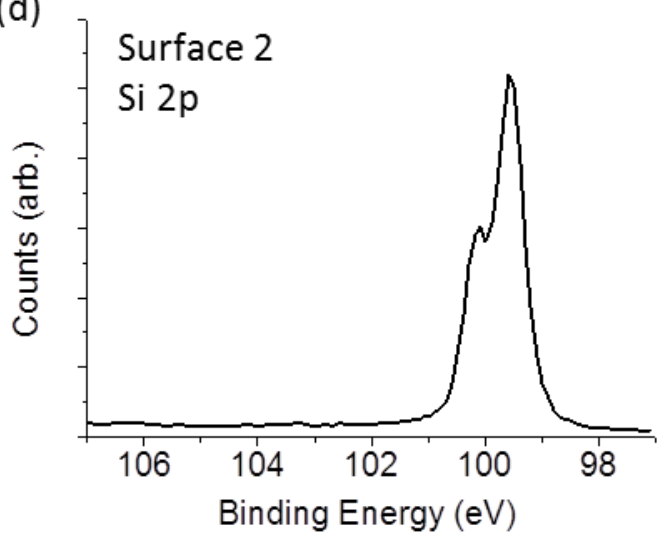

Figure 2. (a) XPS survey scan of "click" azido-OEG-NH 2 functionalized SOS surface 2. High resolution scans for (b) nitrogen, (c) carbon and (d) silicon.

Photolithography on 1, 8-nonadiyne modified SOS/Si substrate. To study the potential effects of photolithography on alkyne monolayers and the effect on "click" reactions, surfaces 3-5 were prepared as described in Scheme 1b. After photolithographic treatment, which involves spin-coating, UV-exposure, development and removal with acetone, the 
contact angle for surface 4 decreased by $\sim 12^{\circ}$ (see Table 1), indicating a change of the surface chemistry. AFM measurements showed an obvious trace of the pattern on surface 3 (Figure 3), indicative of a chemical reaction with compounds in the photoresist. The average height of the exposed layer, determined from the AFM topography, was $\sim 4.7 \AA$, which is consistent with the ellipsometry thickness that shows an increase from $10.6 \AA$ (surface 1) to $15.5 \AA$ (surface 4) (see Table 1). The chemical modification of the surface was also confirmed by XPS measurements (Figure S2, Supporting Information). It was found that the atomic ratios of $\mathrm{C} / \mathrm{Si}$ and $\mathrm{O} / \mathrm{Si}$ on surface 4 had increased by factors of $\sim 1.95$ and $\sim 3.5$, respectively, compared to the results of surface 1 (Figure S2c, Supporting Information). The Si $2 p$ narrow scan showed minor emissions associated with silicon oxide species (102$104 \mathrm{eV}$ ), indicating some oxidized spots were formed in defective regions of the monolayer due to the photolithographic treatment. The area of the oxidized silicon peak was estimated to be $\sim 3 \%$ of the total Si intensity, corresponding to $\sim 0.17$ monolayers of oxidized silicon ${ }^{57}$ (Figure S2b, Supporting Information). Further investigation revealed that the surface oxidation occurred following washing with the developer rather than UV-exposure.
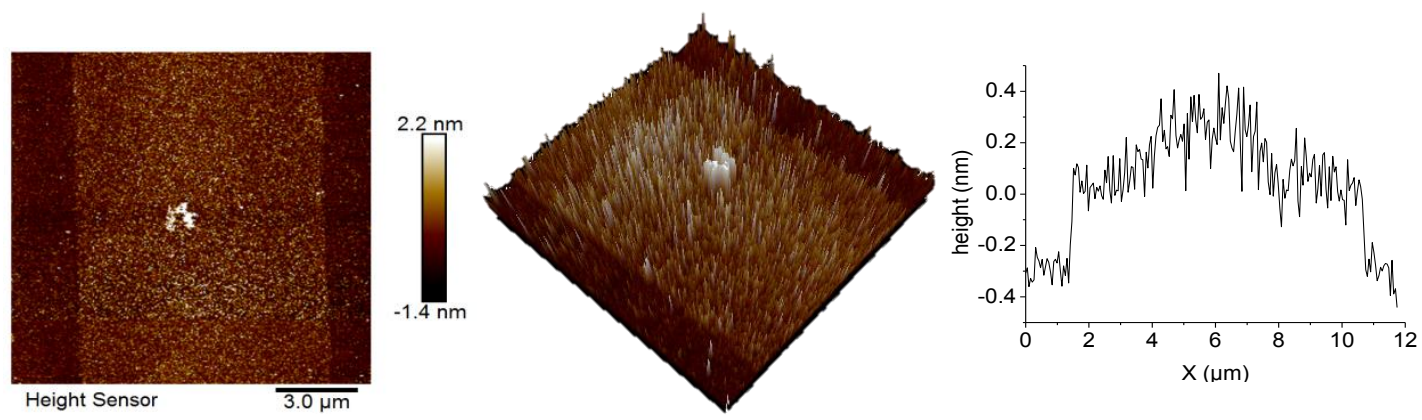

Figure 3. AFM images and topography of 1, 8-nonadiyne modified silicon surface 3 following photolithographic treatment (surface 3, Scheme 1b)

It is established that during exposure to UV light, the photoactive compound diazonaphthaquinone 1 in a positive photoresist (i.e. S1818 or AZ6612 which has been used 
elsewhere ${ }^{6-7}$ ) loses $\mathrm{N}_{2}$ to generate carbene intermediate 2 (Scheme S2, Supporting Information). The carbene transforms into ketene 3, which then forms carboxylic acid $\mathbf{4}$ after reaction with water ${ }^{58}$. The carboxylic acid is then dissolved in the alkaline developer and removed from the surface. However, due to the multi-step nature of the process and the high reactivity of the intermediates, it has been proposed that particulate contamination may be a constant problem in photolithography ${ }^{48}$. Presumably, in this case either carbene 2 or the photochemically generated ketene intermediate $\mathbf{3}$ undergoes partial reaction with the alkyneterminated monolayer, the latter most probably via a [2+2] cycloaddition, resulting in an aromatic-terminated surface.

LAPS has been validated to be a very sensitive technique to detect the properties of surfaces, such as the surface potential, with spatial resolution. Using a $405 \mathrm{~nm}$ laser and SAM modified SOS with a $1 \mu \mathrm{m}$ thick silicon layer, a resolution of $\sim 2.0 \mu \mathrm{m}$ has been obtained ${ }^{33}$. Figure 4a shows the LAPS image of surface 3 measured at $0.55 \mathrm{~V}$, which is identical to the photoresist pattern before its removal with acetone. At the chosen voltage, the photocurrent was slightly smaller on the UV-exposed region (dark strips shown in Figure 4a) than on the non-exposed region. This corresponds to a more negatively charged surface on the area exposed to UV light. Whilst this is an empirical observation, we speculate that the negative charge may be due to two reasons: Firstly, as aromatic compounds readily participate in intramolecular $\pi-\pi$ interactions with neighboring molecules, it is possible, that the indene carboxylic acid (4) has been absorbed onto the surface, resulting in a small negative surface charge in aqueous solution. Alternatively, the minor quantity of oxidized silicon shown by XPS to be formed after the photolithographic treatment (see Figure S2b, Supporting Information) could also contribute to the negative surface charge. Photocurrent curves for the areas on the UV-exposed region and off the UV-exposed region are shown in Figure 4b. The lower part of the photocurrent curve on the UV-exposed region shifted by $+21 \mathrm{mV}$ compared 
to the non-exposed region. The potential shift appearing only in the lower part of $I-V$ curves can be explained by the incomplete coverage of the negatively charged species ${ }^{33}$.

In order to further investigate the nature of the chemistry between the photoresist intermediates and organic monolayers, the same surface treatments were performed on carboxylic acid-terminated and alkyl-terminated monolayers (Scheme S2a, Supporting Information). The detailed procedures for the undecylenic acid and 1-octadecene monolayer modifications on SOS have been described elsewhere ${ }^{33}$. AFM imaging for the surface modified with an undecylenic acid monolayer (surface 9, Supporting Information) showed an increase of surface height by $\sim 6 \AA$ for the UV-exposed region (Figure S3, Supporting Information), again indicating that the carboxylic acid also undergoes unwanted chemical modification. Figure $4 \mathrm{c}$ shows the LAPS image of this surface measured at $0.5 \mathrm{~V}$, which revealed the photocurrent to be greater on the UV-exposed region (bright strips shown in Figure 4c) than that from the unexposed area, which is the opposite of the result observed for the 1, 8-nonadiyne monolayer modified substrate. The corresponding photocurrent curves showed a shift by $-64 \mathrm{mV}$ for the surface exposed with UV light and may be due to the reaction of the carboxylic acid with the ketene, resulting in a less negatively charged surface.

In light of this, we decided to investigate the effect of the photoresist treatment on a saturated hydrocarbon monolayer, which ostensibly contains little chemically reactive functionality. In this case, we again observed a decreased photocurrent in the LAPS image of the UV-exposed areas following treatment with photoresist (see Figure $4 \mathrm{e}$ and $\mathrm{f}$ ) and, as for the 1, 8-nonadiyne modified surface, a lower photocurrent indicated a more negatively charged surface in the exposed areas. As there is unlikely to be any reaction between the monolayer and $\mathbf{3}$ it would appear to indicate that carbene $\mathbf{4}$ has undergone $\mathrm{C}-\mathrm{H}$ bond insertion ${ }^{59}$ and that this results in a similar effect on the surface charge as observed for the alkyne-terminated monolayers. 


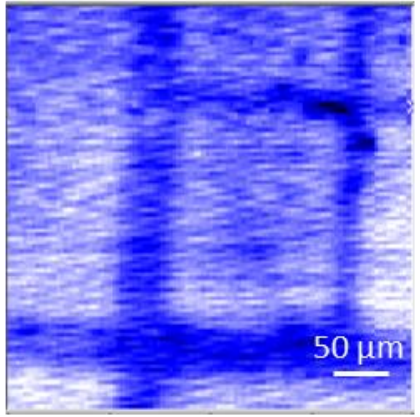

(a)

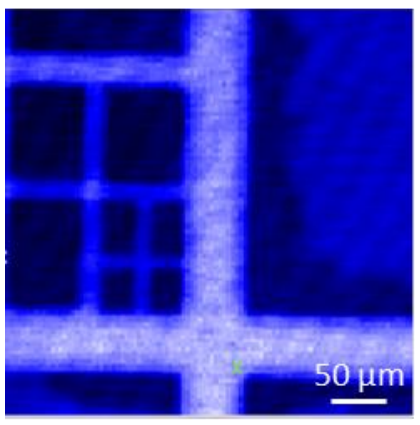

(c)

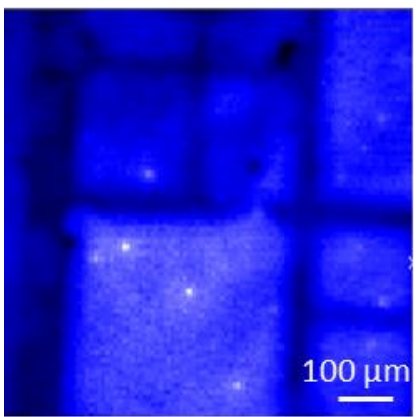

(e)
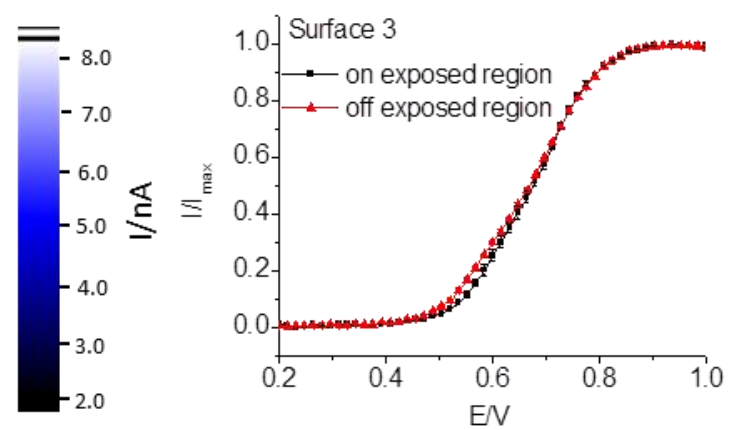

(b)
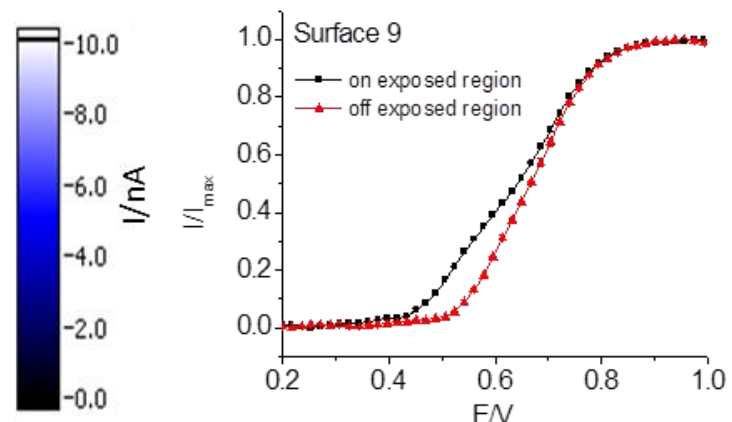

(d)
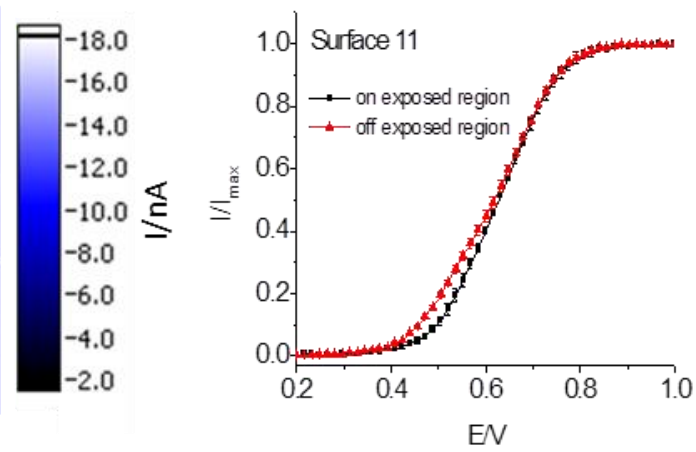

(f)

Figure 4. LAPS images of SOS substrates modified with different organic monolayers following photolithographic treatment: (a) 1, 8-nonadiyne modified surface (surface 3, Scheme 1b) measured at $0.55 \mathrm{~V}$ (dark lines: exposed to UV); (c) undecylenic acid modified SOS (surface 9, Scheme S1, Supporting Information) measured at $0.5 \mathrm{~V}$ (bright lines: exposed to UV) and (e) 1-octadecence modified SOS (surface 11, Supporting Information ) measured at $0.5 \mathrm{~V}$ (dark lines: exposed to UV). Corresponding $I-V$ curves for UV-exposed 
and non-exposed regions on (b) 1, 8-nonadiyne; (d) undecylenic acid and (f) 1-octadecence modified SOS surfaces.

To check the effect of the photolithographic treatment on the efficacy of CuAAC "click" modification, surface 4 was subjected to standard "click" modification with azido-OEG- $\mathrm{NH}_{2}$ to generate surface 5, which was characterized. Both the water contact angle and the ellipsometric thickness changed significantly (see Table 1), showing that "click" modification had occurred to some extent, but these differed significantly from the values seen for surface 7. The XPS survey scan and N 1s scan also corroborated the success of the "click" surface modification (Figure S4, Supporting Information). This indicates that if the terminal alkynes do indeed undergo [2+2] cycloadditions with ketene 3 during UV-exposure that a number remain viable in the CuAAC reaction. However, a lower level of nitrogen incorporation into the surface following the "click" modification of the surface when compared to surface 2, would also be expected. This would be manifested in a reduced N/C atomic ratio observed in the XPS survey scan, which is clearly seen (Figure 5a). Furthermore, the XPS spectrum at 102-104 eV shows high levels of oxidized silicon species (Figure 5b) and the $\mathrm{SiO}_{\mathrm{x}}$ fractional monolayer coverage was estimated to be $\sim 0.32$. This is double the level found following photolithography and indicates that chemical modification via this route results in significant perturbation of the organic monolayer and that it is no longer able to effectively protect the underlying silicon substrate from oxidation. 


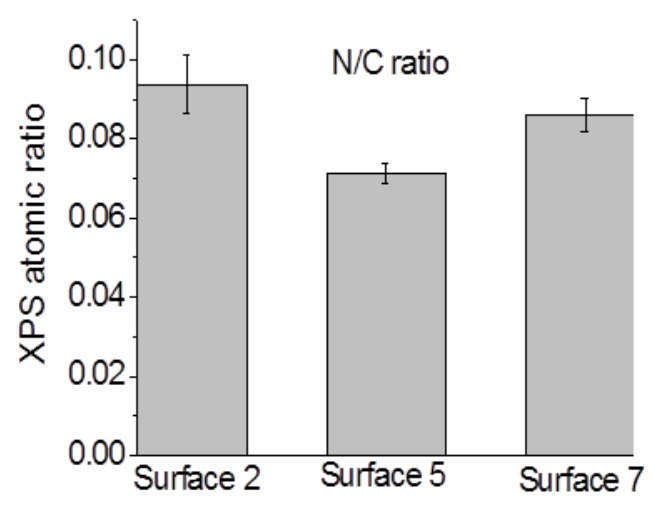

(a)

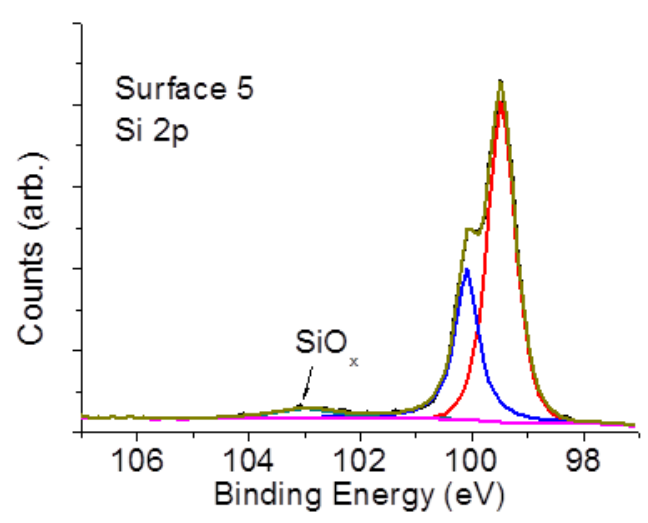

(b)

Figure 5. (a) Atomic ratio (N/C) of surface 2, surface 5 and surface 7 evaluated from XPS surveys scans (b) XPS high resolution $\mathrm{Si} 2 \mathrm{p}$ scan of azido-OEG-NH $\mathrm{H}_{2}$ functionalized SOS substrate after photolithographic treatment (surface 5).

Chemical patterning through the combination of $\mu \mathrm{CP}$ and "click" chemistry. Due to the surface contamination caused by the photolithography, we sought a clean and quick method $\mu \mathrm{CP}$ - to chemically pattern 1,8 -nonadiyne modified SOS or silicon substrates. As shown in Scheme 1c, the azide inks were printed onto the alkyne-terminated SAMs on SOS through "click" reactions to gain surface 6 . To demonstrate that a covalent bond is formed in the nanoscale confinement, a flat featureless PDMS stamp was used to produce a homogeneously functionalized surface (surface 7). The contact angle $\left(46 \pm 2^{\circ}\right)$ and monolayer thickness $(15.8 \AA)$ after the CuAAC “click" reaction via $\mu \mathrm{CP}$ are shown in Table 1 , which are comparable to the results of surface 2. The successful coupling was further confirmed by XPS results (Figure S5, Supporting Information), which show that the $\mathrm{C} / \mathrm{N}$ atomic ratio of surface $7(\sim 0.086)$ is close to the value of surface $2(\sim 0.093)$, indicating a comparable level of "click" triazole incorporation on the surfaces (Figure 5a). Importantly, the reaction time via $\mu \mathrm{CP}$ is significantly shorter than the time using the traditional "click" condition ( $3 \mathrm{~h}$ c.f. $24 \mathrm{~h}$ ). An even shorter reaction time of 15 min as suggested by Ravoo and Reinhoudt ${ }^{9,29}$ was also 
tested. However, both the contact angle of $54.7^{\circ}$ and the nitrogen/carbon ratio of $0.046 \pm 0.0035$ measured by XPS (compared to a nitrogen carbon ratio of $(\sim 0.086 \pm 0.0043$ after $3 \mathrm{~h}$ ) indicated that the longer reaction time of $3 \mathrm{~h}$ was required. Encouragingly, negligible amounts of silicon oxide were observed from the Si $2 p$ narrow scan, demonstrating a non-invasive method to functionalize and pattern the oxide-free silicon surface with SAMs. To validate the combined patterning technique, LAPS was used to image the chemically patterned SOS substrate (surface 6). The LAPS image obtained showed very good agreement with the patterns of the PDMS stamp. At the chosen voltage $(0.44 \mathrm{~V})$, the photocurrent was greater on the "click" functionalized surface (bright islands shown in Figure 6a) than on the 1, 8-nonadiyne monolayer surface, which corresponds to the positive charge introduced by $\mathrm{NH}_{2}$-terminated monolayers. Figure $6 \mathrm{~b}$ shows the $I-V$ curves measured on the circular feature (azido-OEG- $\mathrm{NH}_{2}$ ) and the background region (1,8-nonadiyne). The lower part of the curve on the amino-terminated region shifted by $-50 \mathrm{mV}$ compared to the 1,8 -nonadiyne modified surface, which is slightly greater than the shift caused by physically absorbed poly(allylamine hydrochloride) $(\mathrm{PAH})(-41 \mathrm{mV})^{33}$.

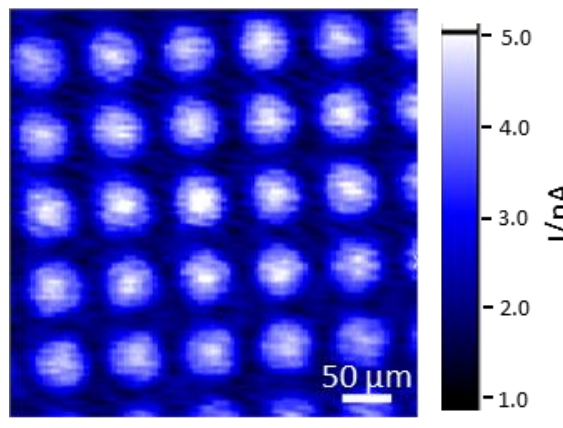

(a)

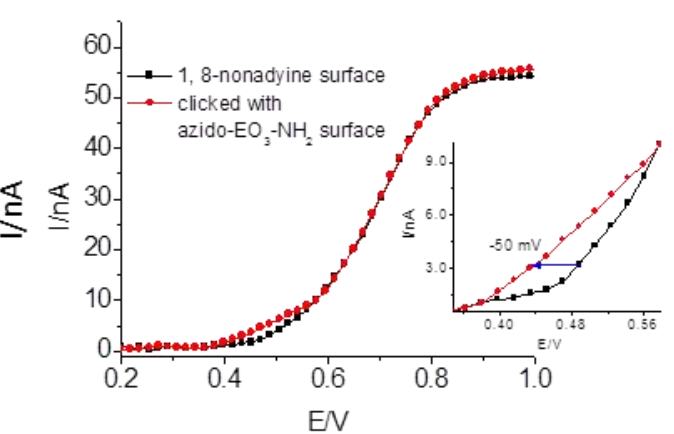

(b)

Figure 6. (a) LAPS image of chemically patterned non-oxide SOS substrate (surface 6) with azido-OEG- $\mathrm{NH}_{2}$ on the circular regions and 1, 8-nonadiyne on the background (measured at $0.44 \mathrm{~V})(\mathrm{b})$ corresponding $I-V$ curves of the circular feature and the background region. 
As a control, inks without azide were printed onto 1, 8-nonadiyne modified substrates using the procedure described in Scheme 1c. For the sample printed with a flat PDMS stamp, the contact angle showed no changes and ellipsometry showed an increase of $1 \AA$, which is within the error of ellipsometry. The sample contacted with a PDMS stamp structured with patterns showed no photocurrent contrast over the entire surface measured at depletion voltages.

\section{Conclusion}

We have demonstrated that $\mu \mathrm{CP}$ followed by "click" chemistry is a mild and efficient means for chemical patterning on oxide-free silicon or SOS surfaces whereas the combination of photolithography and "click" chemistry is not. As evidenced by XPS spectra, approximately $17 \%$ of $\mathrm{SiO}_{\mathrm{x}}$ monolayers were formed after photoresist processing on initially oxide-free SAMs and this value was doubled after further derivatization via a "click" reaction. The surface characterization results and LAPS images demonstrated that side-reactions between the photoactive compounds in the photoresist and SAMs occurred, causing undesirable chemical contamination of the surface. However, $\mu \mathrm{CP}$ was shown to be an effective method to chemically pattern alkyne-terminated monolayers through "click" reactions. The atomic $\mathrm{N} / \mathrm{C}$ ratio was comparable to that of a conventionally formed "click" monolayer, but surface modification was achieved with a much shorter reaction time. More importantly, no evidence of surface contamination with silicon oxide species was observed after $\mu \mathrm{CP}$-induced "click" reactions. The successful chemical patterning was validated using LAPS. The voltage shift ($50 \mathrm{mV}$ ) in the depletion region of the photocurrent curves indicated a good sensitivity for the measurement of the surface charge effects induced by the chemically bonded $\mathrm{NH}_{2}$-terminated molecules. Apart from being suitable for the measurement of surface charge distribution, LAPS was also shown to be extremely sensitive to surface contamination. This will broaden 
the application of LAPS from a sensor technique to a characterization tool for SAM modified silicon substrates.

Acknowledgements. The authors are grateful to the China Scholarship Council for funding (JW, FW and JZ), NEXUS (UK) for XPS measurements; Professor J. Justin Gooding and Ying Yang (The University of New South Wales, Sydney, Australia) for help with the monolayer modification of silicon, Dr Julien Gautrot's Research Group, QMUL, for help with ellipsometry measurements and PDMS stamp preparation.

Supporting Information Available: LAPS set-up and additional sample preparation schedule for surfaces 9 and 11, XPS spectra and AFM results. This material is available free of charge via the Internet at http://pubs.acs.org.

\section{Reference}

1. Lee, C.-S.; Lee, S.-H.; Park, S.-S.; Kim, Y.-K.; Kim, B.-G. Protein patterning on silicon-based surface using background hydrophobic thin film. Biosensors and Bioelectronics 2003, 18 (4), 437-444. 2. Lenci, S.; Tedeschi, L.; Pieri, F.; Domenici, C. UV lithography-based protein patterning on silicon: Towards the integration of bioactive surfaces and CMOS electronics. Applied Surface Science 2011, $257(20), 8413-8419$.

3. Mrksich, M.; Whitesides, G. M. Patterning self-assembled monolayers using microcontact printing: a new technology for biosensors? Trends in biotechnology 1995, 13 (6), 228-235.

4. $\quad$ Smith, R. K.; Lewis, P. A.; Weiss, P. S. Patterning self-assembled monolayers. Progress in Surface Science 2004, 75 (1), 1-68.

5. Sorribas, H.; Padeste, C.; Tiefenauer, L. Photolithographic generation of protein micropatterns for neuron culture applications. Biomaterials 2002, 23 (3), 893-900.

6. Zhu, Y.; Gupta, B.; Guan, B.; Ciampi, S.; Reece, P. J.; Gooding, J. J. Photolithographic Strategy for Patterning Preformed, Chemically Modified, Porous Silicon Photonic Crystal Using Click Chemistry. ACS applied materials \& interfaces 2013, 5 (14), 6514-6521.

7. Zhu, Y.; Soeriyadi, A. H.; Parker, S. G.; Reece, P. J.; Gooding, J. J. Chemical patterning on preformed porous silicon photonic crystals: towards multiplex detection of protease activity at precise positions. Journal of Materials Chemistry B 2014, 2 (23), 3582-3588.

8. Itoga, K.; Kobayashi, J.; Yamato, M.; Kikuchi, A.; Okano, T. Maskless liquid-crystal-display projection photolithography for improved design flexibility of cellular micropatterns. Biomaterials 2006, 27 (15), 3005-3009.

9. Rozkiewicz, D. I.; Jańczewski, D.; Verboom, W.; Ravoo, B. J.; Reinhoudt, D. N. “Click” chemistry by microcontact printing. Angewandte Chemie International Edition 2006, 45 (32), 52925296.

10. Kidambi, S.; Chan, C.; Lee, I. Selective depositions on polyelectrolyte multilayers: Selfassembled monolayers of $\mathrm{m}$-dPEG acid as molecular template. Journal of the American Chemical Society 2004, 126 (14), 4697-4703. 
11. Campos, M. A. C.; Paulusse, J. M. J.; Zuilhof, H. Functional monolayers on oxide-free silicon surfaces via thiol-ene click chemistry. Chemical Communications 2010, 46 (30), 5512-5514.

12. Chen, X.; Zhang, J.; Wang, Z.; Yan, Q. Fabrication of submicron-gap electrodes by silicon volume expansion for DNA-detection. Sensors and Actuators A: Physical 2012, 175, 73-77.

13. Prashanth, G. R.; Goudar, V. S.; Suran, S.; Raichur, A. M.; Varma, M. M. Non-covalent functionalization using lithographically patterned polyelectrolyte multilayers for high-density microarrays. Sensors and Actuators B: Chemical 2012, 171, 315-322.

14. Rozkiewicz, D. I.; Gierlich, J.; Burley, G. A.; Gutsmiedl, K.; Carell, T.; Ravoo, B. J.; Reinhoudt, D. N. Transfer printing of DNA by "click" chemistry. ChemBioChem 2007, 8 (16), 1997-2002.

15. Wang, Z.; Zhang, P.; Kirkland, B.; Liu, Y.; Guan, J. Microcontact printing of polyelectrolytes on PEG using an unmodified PDMS stamp for micropatterning nanoparticles, DNA, proteins and cells. Soft Matter 2012, 8 (29), 7630-7637.

16. Lange, S. A.; Benes, V.; Kern, D. P.; Hörber, J. H.; Bernard, A. Microcontact printing of DNA molecules. Analytical Chemistry 2004, 76 (6), 1641-1647.

17. Marchesan, S.; Easton, C. D.; Styan, K. E.; Leech, P.; Gengenbach, T. R.; Forsythe, J. S.; Hartley, P. G. SU-8 photolithography on reactive plasma thin-films: coated microwells for peptide display. Colloids and Surfaces B: Biointerfaces 2013, 108 (0), 313-321.

18. Hahn, M. S.; Taite, L. J.; Moon, J. J.; Rowland, M. C.; Ruffino, K. A.; West, J. L. Photolithographic patterning of polyethylene glycol hydrogels. Biomaterials 2006, 27 (12), 25192524.

19. Csucs, G.; Michel, R.; Lussi, J. W.; Textor, M.; Danuser, G. Microcontact printing of novel copolymers in combination with proteins for cell-biological applications. Biomaterials 2003, 24 (10), 1713-1720.

20. Sullivan, T. P.; van Poll, M. L.; Dankers, P. Y.; Huck, W. T. Forced peptide synthesis in nanoscale confinement under elastomeric stamps. Angewandte Chemie 2004, 116 (32), 4286-4289. 21. Kolb, H. C.; Finn, M.; Sharpless, K. B. Click chemistry: diverse chemical function from a few good reactions. Angewandte Chemie International Edition 2001, 40 (11), 2004-2021.

22. Rohde, R. D.; Agnew, H. D.; Yeo, W.-S.; Bailey, R. C.; Heath, J. R. A Non-Oxidative Approach toward Chemically and Electrochemically Functionalizing Si(111). Journal of the American Chemical Society 2006, 128 (29), 9518-9525.

23. Ciampi, S.; Le Saux, G.; Harper, J. B.; Gooding, J. J. Optimization of Click Chemistry of Ferrocene Derivatives on Acetylene-Functionalized Silicon(100) Surfaces. Electroanalysis 2008, 20 (14), 1513-1519.

24. Michaels, P.; Alam, M. T.; Ciampi, S.; Rouesnel, W.; Parker, S. G.; Choudhury, M. H.; Gooding, J. J. A robust DNA interface on a silicon electrode. Chemical Communications 2014.

25. Balaur, E.; Djenizian, T.; Boukherroub, R.; Chazalviel, J.; Ozanam, F.; Schmuki, P. Electron beam-induced modification of organic monolayers on $\mathrm{Si}$ (111) surfaces used for selective electrodeposition. Electrochemistry communications 2004, 6 (2), 153-157.

26. Saito, N.; Youda, S.; Hayashi, K.; Sugimura, H.; Takai, O. Chemical resistivity of self-assembled monolayer covalently attached to silicon substrate to hydrofluoric acid and ammonium fluoride. Surface Science 2003, 532, 970-975.

27. Saini, G.; Gates, R.; Asplund, M. C.; Blair, S.; Attavar, S.; Linford, M. R. Directing polyallylamine adsorption on microlens array patterned silicon for microarray fabrication. Lab on $a$ Chip 2009, 9 (12), 1789-1796.

28. Pike, A. R.; Patole, S. N.; Murray, N. C.; Ilyas, T.; Connolly, B. A.; Horrocks, B. R.; Houlton, A. Covalent and Non - covalent Attachment and Patterning of Polypyrrole at Silicon Surfaces. Advanced Materials 2003, 15 (3), 254-257.

29. Mehlich, J.; Ravoo, B. J. Click chemistry by microcontact printing on self-assembled monolayers: A structure-reactivity study by fluorescence microscopy. Organic \& Biomolecular Chemistry 2011, 9 (11), 4108-4115. 
30. Li, Y.; Cai, C. Click Chemistry-Based Functionalization on Non-Oxidized Silicon Substrates. Chemistry-an Asian Journal 2011, 6 (10), 2592-2605.

31. Aureau, D.; Rappich, J.; Moraillon, A.; Allongue, P.; Ozanam, F.; Chazalviel, J. N. In situ monitoring of the electronic properties and the $\mathrm{pH}$ stability of grafted $\mathrm{Si}(1 \& \# x a 0 ; 1 \& \# x a 0 ; 1)$. Journal of Electroanalytical Chemistry 2010, 646 (1-2), 33-42.

32. Kehr, N. S.; El-Gindi, J.; Galla, H.-J.; De Cola, L. Click chemistry on self-assembled monolayer of zeolite $\mathrm{L}$ crystals by microcontact printing - Applications in nanobiotechnology. Microporous and Mesoporous Materials 2011, 144 (1-3), 9-14.

33. Wang, J.; Zhou, Y.; Watkinson, M.; Gautrot, J.; Krause, S. High-sensitivity light-addressable potentiometric sensors using silicon on sapphire functionalized with self-assembled organic monolayers. Sensors and Actuators B: Chemical 2015, 209, 230-236.

34. Krause, S.; Moritz, W.; Talabani, H.; Xu, M.; Sabot, A.; Ensell, G. Scanning photo-induced impedance microscopy-resolution studies and polymer characterization. Electrochimica Acta 2006, $51(8), 1423-1430$.

35. Seki, A.; Kawakubo, K.; Iga, M.; Nomura, S. Microbial assay for tryptophan using siliconbased transducer. Sensors and Actuators B: Chemical 2003, 94 (3), 253-256.

36. Yoshinobu, T.; Ecken, H.; Md. Ismail, A. B.; Iwasaki, H.; Lüth, H.; Schöning, M. J. Chemical imaging sensor and its application to biological systems. Electrochimica Acta 2001, 47 (1-2), 259-263. 37. Gehring, A. G.; Patterson, D. L.; Tu, S.-I. Use of a Light-Addressable Potentiometric Sensor for the Detection ofEscherichia coliO157:H7. Analytical Biochemistry 1998, 258 (2), 293-298.

38. Stein, B.; George, M.; Gaub, H. E.; Behrends, J. C.; Parak, W. J. Spatially resolved monitoring of cellular metabolic activity with a semiconductor-based biosensor. Biosensors and Bioelectronics 2003, 18 (1), 31-41.

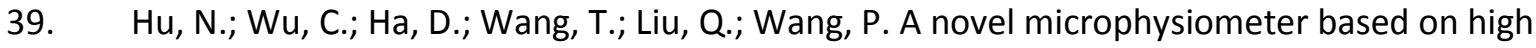
sensitivity LAPS and microfluidic system for cellular metabolism study and rapid drug screening. Biosensors and Bioelectronics 2013, 40 (1), 167-173.

40. Du, L.; Zou, L.; Zhao, L.; Huang, L.; Wang, P.; Wu, C. Label-free functional assays of chemical receptors using a bioengineered cell-based biosensor with localized extracellular acidification measurement. Biosensors and Bioelectronics 2014, 54 (0), 623-627.

41. Liu, Q.; Yu, H.; Tan, Z.; Cai, H.; Ye, W.; Zhang, M.; Wang, P. In vitro assessing the risk of druginduced cardiotoxicity by embryonic stem cell-based biosensor. Sensors and Actuators B: Chemical 2011, 155 (1), 214-219.

42. Du, L.; Wu, C.; Peng, H.; Zhao, L.; Huang, L.; Wang, P. Bioengineered olfactory sensory neuron-based biosensor for specific odorant detection. Biosensors and Bioelectronics 2013, 40 (1), 401-406.

43. Ng, A.; Ciampi, S.; James, M.; Harper, J. B.; Gooding, J. J. Comparing the Reactivity of Alkynes and Alkenes on Silicon (100) Surfacest. Langmuir 2009, 25 (24), 13934-13941.

44. James, M.; Ciampi, S.; Darwish, T. A.; Hanley, T. L.; Sylvester, S. O.; Gooding, J. J. Nanoscale water condensation on click-functionalized self-assembled monolayers. Langmuir 2011, 27 (17), 10753-10762.

45. Ciampi, S.; Böcking, T.; Kilian, K. A.; James, M.; Harper, J. B.; Gooding, J. J. Functionalization of Acetylene-Terminated Monolayers on Si(100) Surfaces: A Click Chemistry Approach. Langmuir 2007, 23 (18), 9320-9329.

46. Liu, H.; Duclairoir, F.; Fleury, B.; Dubois, L.; Chenavier, Y.; Marchon, J.-C. Porphyrin anchoring on Si(100) using a [small beta]-pyrrolic position. Dalton Transactions 2009, (19), 3793-3799.

47. Ciampi, S.; Eggers, P. K.; Le Saux, G.; James, M.; Harper, J. B.; Gooding, J. J. Silicon (100) Electrodes Resistant to Oxidation in Aqueous Solutions: An Unexpected Benefit of Surface Acetylene Moieties. Langmuir 2009, 25 (4), 2530-2539.

48. Xu, F.; Xu, D.; Kang, E.; Neoh, K. Self-doped conductive polymer-silicon hybrids from atom transfer radical graft copolymerization of sodium styrenesulfonate with polyaniline covalently tethered on the Si (100) surface. Journal of Materials Chemistry 2004, 14 (17), 2674-2682. 
49. Gautrot, J. E.; Trappmann, B.; Oceguera-Yanez, F.; Connelly, J.; He, X.; Watt, F. M.; Huck, W. $T$. Exploiting the superior protein resistance of polymer brushes to control single cell adhesion and polarisation at the micron scale. Biomaterials 2010, 31 (18), 5030-5041.

50. Chen, L.; Zhou, Y.; Jiang, S.; Kunze, J.; Schmuki, P.; Krause, S. High resolution LAPS and SPIM. Electrochemistry Communications 2010, 12 (6), 758-760.

51. Ciampi, S.; James, M.; Michaels, P.; Gooding, J. J. Tandem "click" reactions at acetyleneterminated Si (100) monolayers. Langmuir 2011, 27 (11), 6940-6949.

52. Shamsi, F.; Coster, H.; Jolliffe, K. A. Characterization of peptide immobilization on an acetylene terminated surface via click chemistry. Surface Science 2011, 605 (19-20), 1763-1770.

53. Ng, C. C. A.; Ciampi, S.; Harper, J. B.; Gooding, J. J. Antifouling behaviour of silicon surfaces modified with self-assembled monolayers containing both ethylene glycol and charged moieties. Surface Science 2010, 604 (17), 1388-1394.

54. Palacin, T.; Khanh, H. L.; Jousselme, B.; Jegou, P.; Filoramo, A.; Ehli, C.; Guldi, D. M.; Campidelli, S. Efficient functionalization of carbon nanotubes with porphyrin dendrons via click chemistry. Journal of the American Chemical Society 2009, 131 (42), 15394-15402.

55. Wei, P. J.; Yu, G. Q.; Naruta, Y.; Liu, J. G. Covalent Grafting of Carbon Nanotubes with a Biomimetic Heme Model Compound To Enhance Oxygen Reduction Reactions. Angewandte Chemie International Edition 2014, 53 (26), 6659-6663.

56. Ciampi, S.; Gooding, J. J. Direct electrochemistry of cytochrome c at modified Si (100) electrodes. Chemistry-A European Journal 2010, 16 (20), 5961-5968.

57. Haber, J. A.; Lewis, N. S. Infrared and X-ray photoelectron spectroscopic studies of the reactions of hydrogen-terminated crystalline $\mathrm{Si}(111)$ and $\mathrm{Si}(100)$ surfaces with $\mathrm{Br} 2,12$, and ferrocenium in alcohol solvents. The Journal of Physical Chemistry B 2002, 106 (14), 3639-3656. 58. Monbouquette, H. G.; Garcia-Garibay, M. Surface nanopatterning. Google Patents, 2004. 59. Smith, M. B. In Organic Synthesis (Third Edition), Smith, M. B., Ed.; Academic Press: Oxford, 2010. 
TOC picture:
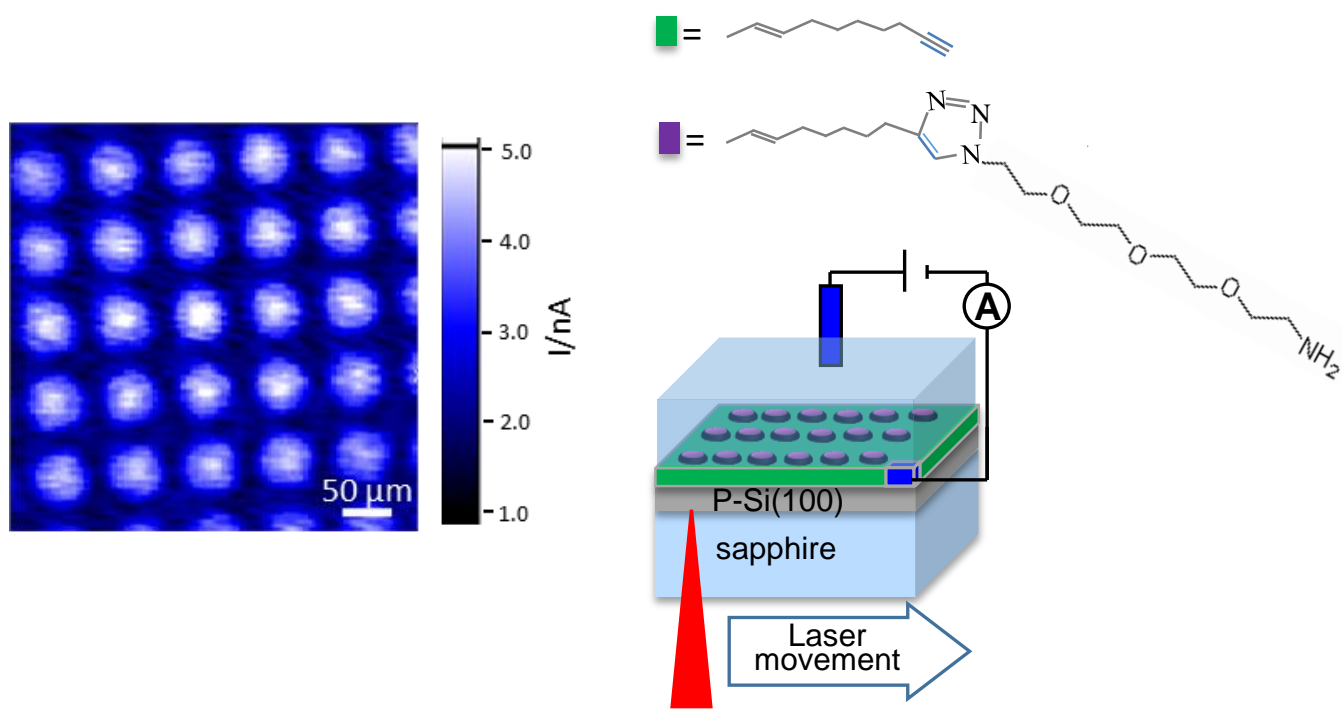